\title{
Investigation of heat waves based on the most typical fuzzy expected values with corresponding trapezoidal membership functions.
}

\author{
Tatiana Kiseliova, ${ }^{1}$ Marina Fandoeva ${ }^{1}$ Anna Sikharulidze ${ }^{1}$ \\ ${ }^{1}$ Iv. Javakhishvili Tbilisi State University, Faculty of Exact and Natural Sciences, University St.2, 0145 Tbilisi,Georgia
}

\begin{abstract}
In this paper we continue to investigate the important parameter of global warming - a heat wave - that has no unique definition and represents the constellation of different factors such as e.g., heat index (temperature + humidity), time periods of excessive heat, etc. We find typical characteristics of heat indexes during different time periods, using most typical fuzzy expected values. Trapezoidal fuzzy sets are used to represent that characteristics. The results are compared with previous work based on the corresponding step-wise functions. We use the data collected in the Ministry of Preservation of the Environment of Georgia during 1955-1970 and 19902007.
\end{abstract}

Keywords: heat index, heat wave, fuzzy expected value.

\section{Introduction: heat index and heat waves}

\subsection{Heat index}

The global warming is a well-known problem that has a great impact on human health. Among several parameters that allow to estimate this effect a heat index (HI) is often used. HI is usually defined as a combination of air temperature and air humidity. HI determines the human-perceived equivalent temperature. There are several ways to estimate HI: by special formula (for example, Steadman [29]) or by special tables where empirical data are collected.

\subsection{Heat waves}

A heat wave notion is closely related to HI. In a general sense a heat wave (HW) can be defined as a prolonged period of excessive heat. Similarly to the description of $\mathrm{HI}$ there is no universal and rigorous descriptions of HW: subjectiveness and uncertainty is a part of existing definitions. We list some examples below.

\subsection{Different approaches to investigate heat waves}

Recently, continuing reports of human mortality because of the effects brought on by extreme heat events worldwide support the necessity for research of HWs. However, defining HW as a hazard is not universal over space because of the differences in climate norms and different types of uncertainty in the estimations.
American Meteorology Society [3] defines HW as a period of abnormally and uncomfortably hot and unusually humid weather. Typically HW lasts two or more days.

In [4] HW is defined as consecutive days with temperature above $30^{\circ} \mathrm{C}$.

The ICARO Project [2] defines HW by means of a temperature threshold of $32^{\circ} \mathrm{C}$ combined with a minimum duration of 2 days. This definition is similar to a definition used in the United States: $90^{\circ} \mathrm{F}\left(32.2^{\circ} \mathrm{C}\right)$ and a minimum duration of 2 days.

There appears to be no official Australian definition of HW [2]. The difficulty in defining HW in Australia has been in establishing an appropriate HI with an acceptable event threshold and duration, and relating it to the climatology of the area under investigation. Various heat or thermal comfort indices have been developed to evaluate heat-related stress (e.g., Temperature Humidity Index [33]), combining air temperature, humidity, and, additionally, in some cases, wind, direct sunlight and nighttime recovery. Two of the most widely used indices in Australia are the apparent temperature (work of Steadman [29]) and the Relative Strain Index, derived by H.S. Belding and T. F. Hatch [6] and discussed in the Goldfields-Eucla climatic survey [1].

Temperatures that would indicate whether a specific location was under the effect of HW would be in the top $5 \%$ for a continuous three-day period. The United States National Weather Service uses the measure of apparent temperature, which is also based on the work of Steadman [29], [30].

In [27] $\mathrm{HW}$ was defined as a period of at least 48 hours during which neither the overnight low nor the daytime high HI falls below the National Weather Service heat stress thresholds $\left(40^{\circ} \mathrm{C}\right.$ and $105^{\circ} \mathrm{F}$, respectively), except at stations for which more than $1 \%$ of both the annual high and low HI observations exceed these thresholds, in which case the $1 \%$ values are used as HW thresholds.

In the work [7] the author defines the notion of HW based on a heat stress. In turn, a heat stress is based on the 24-hour period estimations of HI, and is represented by a value that identifies by summed heat values above $32.2^{\circ} \mathrm{C}\left(90^{\circ} \mathrm{F}\right)$ and $40.6^{\circ} \mathrm{C}\left(105^{\circ}\right)$ subtracted the number of hours of recorded thresholds $\left(32.2^{\circ} \mathrm{C}\left(90^{\circ} \mathrm{F}\right)\right.$, $40.6^{\circ} \mathrm{C}\left(105^{\circ} \mathrm{F}\right)$ and above $)$ times these thresholds. Then accumulated values (Table 1) describe five Categories of a heat stress: each day is assigned a number from 0 to 5, 
0 means no stress (Category 0 is not included into Table 1). In order for HW to be identified, consecutive days must be classified as Category 1 or higher. To classify each HW as individual hazards, the logical solution was to assign the highest value from recorded values of heat stress during the consecutive days. Named as an event, HW with daily categories listed as 1-2-2-3-2-2, for example, would be a 6-day event registering as Category 3 HW.

\begin{tabular}{|l|l|l|l|l|}
\hline & $\begin{array}{l}\text { Category of } \\
\text { a heat stress }\end{array}$ & $\mathrm{HI}-90^{\circ} F$ & $\mathrm{HI}-105^{\circ} F$ & Recovery \\
\hline 1 & Minor & $\geq 40$ & - & - \\
\hline 2 & Moderate & $\geq 80$ & $\geq 1$ & $\leq 10$ \\
\hline 3 & Strong & $\geq 120$ & $\geq 10$ & $\leq 6$ \\
\hline 4 & Severe & $\geq 160$ & $\geq 20$ & $\leq 2$ \\
\hline 5 & Extreme & $\geq 260$ & $\geq 35$ & 0 \\
\hline
\end{tabular}

Table 1: The daily heat stress classification system in Fahrenheit [7].

\subsection{Why it is important to define a heat wave properly?}

Extreme heat is a hazard that is capable of causing economic problems and potentially high mortality rates across several regions simultaneously [7]. We should be able to define what does it mean that a period of time was undertaken by HWs or, in spoken words, that some days, weeks or even months were extremely hot or very hot. This definition can not be based on the value of one HI (for example, in a day), because the frequency, magnitude and duration of the HIs as well as day-to-day magnitude variability, overall frequency of HIs, seasonal timing of events are important characteristics to define HW. Thus, the problem is to define how hot was this or another period of time, which criteria to choose: maximum value of HIs, or the number of days with a high $\mathrm{HI}$, or an intensiveness, or a relativity of HIs, or may be something else?

To combine different parameters as well as to capture above mentioned subjectiveness and imprecision in perception of a heat we propose a new methodology. We used methods based on fuzzy set theory. We chose the method of fuzzy expected value (FEV) [14], [28], [35].

Roughly our new methodology can be described as follows: we ask experts to define "hot weather" and a corresponding fuzzy set. Then we calculate, using real values of HI, the most typical value of the fuzzy set for these values during a particular period, say August (notice, that shorter or longer period can be taken as well). Our results allow to say that this month was extremely hot, very hot, hot, very warm, warm or normal. If obtained characteristics are "very hot" or "extremely hot", we conclude that this month was impact by a severe HW or an extreme HW. Analogically, "hot" corresponds to strong, "very warm", corresponds to a moderate HW and "warm" to a mild HW (see Table 2). Notice, that linguistic terms normal, warm, very warm, hot, very hot, extremely hot we use to deal with HIs, they are defined as shown in Figure 8, whereas words mild, moderate, strong, severe, extreme are applied for HWs. We use different sets of linguistic words to be able to differ two notions: $\mathrm{HI}$ and $\mathrm{HW}$.

\begin{tabular}{|l|l|}
\hline Heat index & Heat wave \\
\hline normal & - \\
\hline warm & mild \\
\hline very warm & moderate \\
\hline hot & strong \\
\hline very hot & severe \\
\hline extremely hot & extreme \\
\hline
\end{tabular}

Table 2: A correspondence between typical characteristics of a heat index and a heat wave.

Different characteristics of HI (e.g., frequency, magnitude and duration) are included in the most typical values. The most typical values allow to see tendency of appearing of HWs in Georgia and it can be used for prognosis in the future. Notice, that we illustrated our approach on the data from a long time-period available for Georgia. But due to its flexibility, our methodology can be easily applied for other regions where measured data are available and experts work out the same problems.

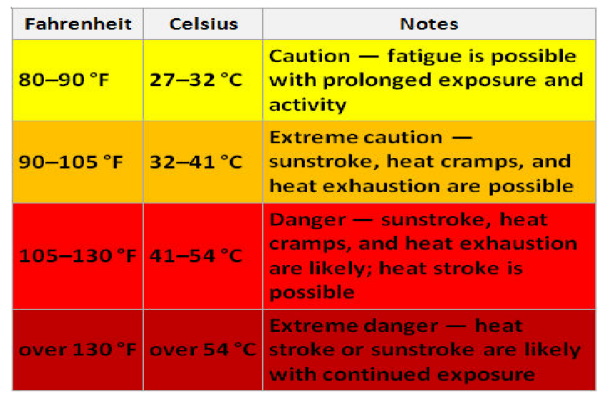

Table 3: Effects of the heat index (shade values).

The paper is organized as follows. In Section 2 we shortly describe the data available for investigation, how they are organized and how HI is calculated. Notice, that we use the meteorological data of Georgia. In Section $3 \mathrm{FEV}$ approach is applied to our problem. In Section 4 we compare the previously done investigations based on the step-wise membership functions with actual trapezoidal ones. In Section 5 we compare the existing methodologies and approaches with our investigation. The final remarks and conclusions are collected in Section 6.

\section{Heat index in Georgia}

A simple dependence between air temperature and air humidity does not exist. In our investigation we have used the calculations based on the work of G. Steadman [29]: the author proposed a formula for approximating 


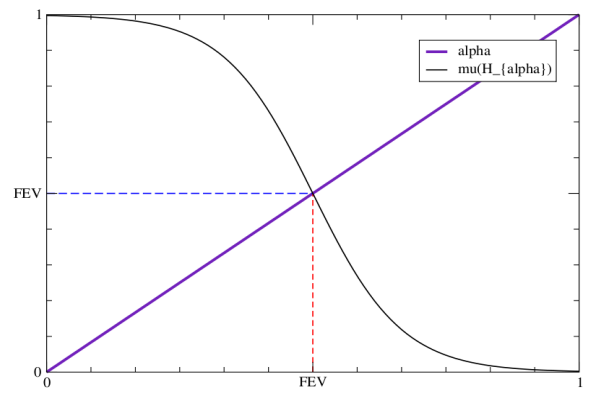

Figure 1: A geometric interpretation of FEV for the continuous function $\mu\left(H_{\alpha}\right), \alpha \in[0,1]$.

the HI in degrees Fahrenheit. This formula is useful only when the air temperature is at least $80^{\circ} \mathrm{F}$ and the relative humidity is at least $40 \%$ (this is a case for Georgia):

$$
\begin{gathered}
H I=c_{1}+c_{2} T+c_{3} R+c_{4} T R+c_{5} T^{2}+c_{6} R^{2}+c_{7} T^{2} R+ \\
+c_{8} T R^{2}+c_{9} T^{2} R^{2}
\end{gathered}
$$

where $H I$ is a heat index (in degrees Fahrenheit); $\mathrm{T}$ is an ambient temperature (in degrees Fahrenheit); $\mathrm{R}$ is a relative humidity (in percent); $c_{1}=-42.379 ; c_{2}=$ $2.04901523 ; c_{3}=10.14333127 ; c_{4}=-0.22475541$; $c_{5}=-6.83783 \times 10^{-3} ; c_{6}=-5.481717 \times 10^{-2} ; c_{7}=$ $1.22874 \times 10^{-3} ; c_{8}=8.5282 \times 10^{-4} ; c_{9}=-1.99 \times$ $10^{-6}$. The results of $\mathrm{HI}$ calculation for Tbilisi in $\mathrm{Au}-$

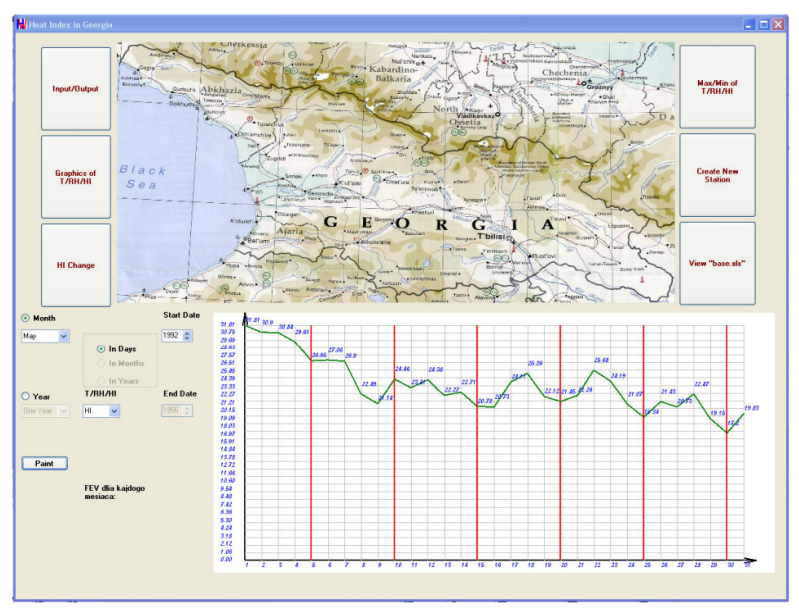

Figure 2: A screening of the front-page of the program with heat indexes on the low part of it.

gust are presented in Figure 2 (down the map).

Investigations of HI are mainly carried out in the following directions:

- influence of HI onto human health;

- HI prognoses in countries under global warming circumstances.

For example, impacts of HI (shade values), published on the website of the Pueblo, CO United States National Weather Service, are presented in Table 3.

Methods used for HI estimations and predictions, usually depend on data available. From this point, data collected in Georgia are immense and allow a wide spectrum of experiments.
We have investigated these empirical data and results of our investigation are presented in this paper. We have deduced typical characteristics of $\mathrm{HI}$ for each month (for available data) and on its base have concluded about HWs in Georgia.

Observations of the global warming influence on the climate change have been started in Georgia since middle 50th [34]. Since that time empirical data are collected. We chose information about air temperature and air humidity collected in four main cities of Georgia: Tbilisi, Poti, Lentekhi, Dedoplistskharo during the two periods (1955-1970 and 1990-2007), and only five months are taken into consideration (May, June, July, August, September). These data are a privilege of Ministry of Preservation of the Environment of Georgia and they have been used for preparation of the Second Communication of Georgia to the United Nations Framework Convention on Climate Change (UNFCCC).

A special program was written to use these data flexibly. It means the data can be visualised graphically or in the form of tables, and different manipulations - such as automatically calculation of HI of each month, each year, each period; finding minimal and maximal values of HIs of a period, etc., - are implemented (e. g., see Figure 2).

\section{Fuzzy expected value}

Having $\mathrm{HI}$ at hand, a question is how to characterise a period of time (week, month, etc.) - hot or not hot, normal, very hot and so on - thus, to give characteristics that used by people for estimations. Based on these characteristics we can estimate a tendency of HI developing in Georgia and, the next step, to estimate an influence of HWs on the human health.

One can use a maximal value of HI during the period, or a number of days with a high HI, relative frequencies of HIs and some criteria else. The difficulty is that each of these characteristics alone do not reflect the influence of $\mathrm{HI}$ on the human health in all complexity.

To capture the majority of the HI characteristics an approach based on fuzzy sets have been chosen. For an estimation we have taken the fuzzy expected value approach [14], [35].

The notion of a fuzzy expected value (FEV) is closely related to the Sugeno integral [31] for a fuzzy set with 
respect to a fuzzy measure. The FEV indicates the most "typical" grade of the membership in a given fuzzy set and the corresponding element from the universe of discourse [14], [28].

Definition 1[11] Let $X$ be a non-empty set, $\mathscr{X}$ be a Borel field ( $\sigma$-algebra) defined on $X$. A fuzzy measure $\mu$ defined on the measurable space $(X, \mathscr{X})$ is a set function $\mu: \mathscr{X} \rightarrow[0,1]$ verifying the following axioms:

1. $\mu(\emptyset)=0 ; \mu(X)=1$;

2. if $A, B \subset \mathscr{X}, A \subset B$ then $\mu(A)<\mu(B)$;

3. if $\left\{A_{k} \mid 1<k<\infty\right\}$ is a monotone sequence, $\forall A_{k} \in$ $\mathscr{X}$, then $\lim _{k \rightarrow \infty} \mu\left(A_{k}\right)=\mu\left(\lim _{k \rightarrow \infty} A_{k}\right)$

$(X, \mathscr{X}, \mu)$ is said to be a fuzzy measure space.

Let $A: X \rightarrow[0,1]$ be the $\mathscr{X}$ measurable function, i.e., $\forall \alpha \in[0,1]: H_{\alpha}=\{x \in X \mid A(x)>\alpha\} \in \mathscr{X} ; \wedge, \vee$ denote min, max, respectively.

Definition 2 The FEV of the fuzzy set $A$ with respect to the fuzzy measure $\mu$ is the Sugeno integral over $X$ :

$$
F E V(A)=d_{d e f} \int_{X} A \circ \mu=\operatorname{def} \sup _{\alpha \in[0,1]}\left\{\alpha \wedge \mu\left(H_{\alpha}\right)\right\}
$$

It follows [28] that FEV somehow "averages" the values of the function $A$ not in the sense of statistical average, but by cutting subsets of the $\alpha$ level, whose values of a fuzzy measure $\mu$ are either sufficiently "high" or sufficiently "low" (see, for example, Figure $1, \mu\left(H_{\alpha}\right)$ is a continuous function, $\alpha \in[0,1])$.

Definition 3 Let $\mu$ be a fuzzy measure on $X=$ $\left\{x_{1}, x_{2}, \ldots, x_{n}\right\}$ (discrete case), $A: X \rightarrow[0,1], X_{i}=$ $\left\{x_{i}, \ldots, x_{n}\right\}$. Let membership degrees be ordered as follows: $A\left(x_{(1)}\right) \leq A\left(x_{(2)}\right) \leq \cdots \leq A\left(x_{(n)}\right)$. Let $\mu$ be a fuzzy measure defined over the subsets of $X$. Then the fuzzy expected value FEV of $A$ is defined as follows

$$
F E V\left(A\left(x_{1}\right), \ldots, A\left(x_{n}\right)\right)=\operatorname{def} \bigvee_{i=1}^{n}\left(A\left(x_{(i)}\right) \wedge \mu\left(X_{i}\right)\right\}
$$

Remark. The $F E V(A)$ may be calculated as the median of the set, ordered from low to high values, $\left\{A\left(x_{(1)}\right), A\left(x_{(2)}\right), \ldots, A\left(x_{(n)}\right), \mu\left(X_{1}\right), \ldots \mu\left(X_{m-1}\right)\right\}$, where $\mu\left(X_{i}\right)=\frac{1}{N} \sum_{j=i+1}^{m} n_{j}, 1 \leq i \leq m-1, N=\sum_{i=1}^{m} n_{i}$ and $n_{i}$ is a finite population.

For more details about FEV a reader is referred to the corresponding sources [10], [14].

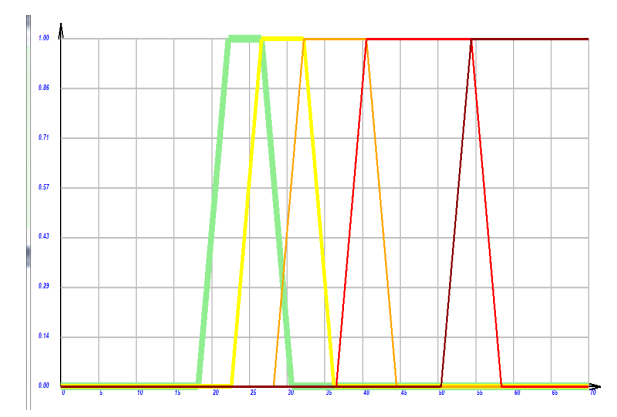

Figure 3: Trapezoidal fuzzy sets for HI charecterisation.
Let us consider an example. Let HI (in Celsius) each day a month (Figure 2) be given. A fuzzy sets normal, warm, very warm, hot, very hot, extremely hot is given as shown in Figure 3. Using fuzzy sets normal, warm, very warm, hot, very hot, extremely hot each HI of a day is assigned a membership degree in the fuzzy sets normal, warm, very warm, hot, very hot, extremely hot.

\begin{tabular}{|l|l|l|l|}
\hline Group & $\begin{array}{l}\text { Support } \\
\text { Interval }\end{array}$ & $\begin{array}{l}\text { Number } \\
\text { of HIs with } \\
\text { values from Int }\end{array}$ & $\alpha$ \\
\hline normal & $<18.2$ & 0 & 0.0 \\
\hline warm & {$[18.2,30.6]$} & 17 & 0.2 \\
\hline $\begin{array}{l}\text { very } \\
\text { warm }\end{array}$ & {$[24.6,36.2]$} & 10 & 0.4 \\
\hline hot & {$[32.2,40.5)$} & 3 & 0.5 \\
\hline $\begin{array}{l}\text { very } \\
\text { hot }\end{array}$ & {$[40.5,54.4)$} & 1 & 0.6 \\
\hline $\begin{array}{l}\text { extremely } \\
\text { hot }\end{array}$ & $\geq 54.4$ & 0 & 1.0 \\
\hline
\end{tabular}

Table 4: Data from Figure 2 for Tbilisi, August 1996, presented in a tabular form.

In the Table 4 the first column is a linguistic name of a fuzzy set : normal, warm, very warm, hot, very hot, extremely hot; the second column shows a support interval for each fuzzy set; the third column shows the number of non-zero HIs from the corresponding support interval; the forth column represents $\alpha$ values in $\alpha$-cuts. Let us call the elements from the last column thresholds. As can be seen, we have five different thresholds: $0.22,0.45,0.56,0.64,1.0$. The Table 5 and Table 6 show the number of HIs with membership values above or equal to each threshold for fuzzy sets warm and very warm and corresponding FEVs:

\begin{tabular}{|l|l|l|l|l|}
\hline Group & Interval & $\begin{array}{l}\text { Thre- } \\
\text { sholds }\end{array}$ & $\begin{array}{l}\text { Num. of HIs } \\
\text { with memb. v. } \\
\text { above thresh. }\end{array}$ & $\begin{array}{l}\text { F } \\
\text { E } \\
\text { V }\end{array}$ \\
\hline warm & {$[18.2,30.6]$} & 0.0 & 31 & \\
& & 0.2 & 28 & \\
& & 0.4 & 24 & 0.6 \\
& & 0.5 & 23 & \\
& & 0.6 & 20 & \\
\hline
\end{tabular}

Table 5: FEV for warm

Analogously the FEVs for very warm, hot, very hot, extremely hot are calculated and all FEVs are represented in Figure 4.

Let us describe the process of FEV calculation in more details for the fuzzy set warm. In the process of finding FEV for the fuzzy set warm for this month, we should check how many fuzzified HIs are above each threshold in percentage terms, (2)), $N$ is the number of all days, in our case $N=31$. From the data available 


\begin{tabular}{|l|l|l|l|l|}
\hline Group & Interval & $\begin{array}{l}\text { Thre- } \\
\text { sholds }\end{array}$ & $\begin{array}{l}\text { Num. of HIs } \\
\text { with memb. v. } \\
\text { above thresh. }\end{array}$ & $\begin{array}{l}\text { F } \\
\text { E } \\
\text { V }\end{array}$ \\
\hline very & {$[24.6,36.2]$} & 0.0 & 31 & \\
warm & & 0.2 & 26 & \\
& & 0.4 & 24 & 0.7 \\
& & 0.5 & 23 & \\
& & 0.6 & 22 & \\
\hline
\end{tabular}

Table 6: FEV for very warm

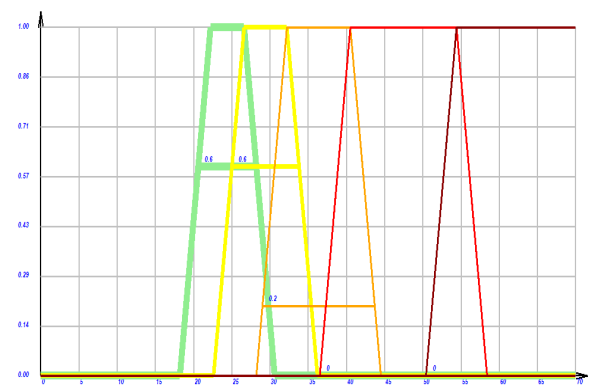

Figure 4: Trapezoidal fuzzy sets with corresponding FEVs.

we have calculated that 31 days (or times) fuzzified $\mathrm{HI}$ is above or equal to $0.0 ; 26$ days $\mathrm{HI}$ is above or equal to $0.2 ; 24$ days $\mathrm{HI}$ is above or equal to $0.4 ; 23$ days $\mathrm{HI}$ is above or equal to $0.5 ; 20$ days $\mathrm{HI}$ is above or equal to 0.6 ; exactly 9 days above 1 for the fuzzy set warm. Pairing the data and rearranging it by increasing order of the membership grade, due to (2), we obtain the following six pairs:

Now, the minimum value of each pair is $\min (0 ; 1)=0$; $\min (0.2 ; 0.84)=0.2 ; \quad \min (0.4 ; 0.77)=0.4$; $\min (0.5 ; 0.74)=0.5 ; \quad \min (1 ; 0.58)=0.58$ and, therefore, following (2), the FEV for the fuzzy set warm, which is the maximum of all these minima, is

$$
\max (0.2 ; 0.4 ; 0.5 ; 0.6 ; 0.58)=0.6(\text { see Figure } 5)
$$

At Figure 6 examples of FEVs for Tbilisi in August (for periods 1955-1970 and 1990-2007) are presented.

\section{Some remarks concerning the fuzzy sets and their corresponding FEVs}

It is important, that for different regions the appropriate membership functions have to be constructed. Because what is warm in one region, for example, can be normal

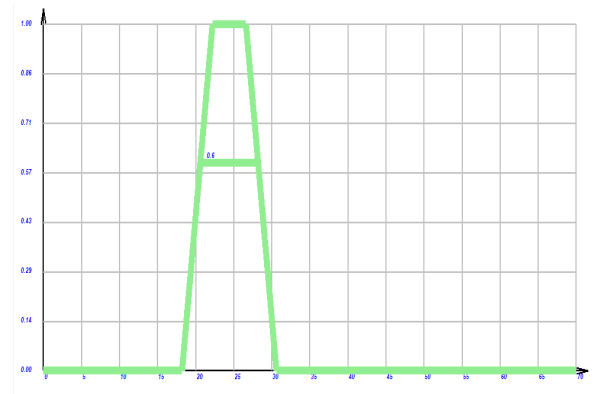

Figure 5: FEV for the fuzzy set warm.

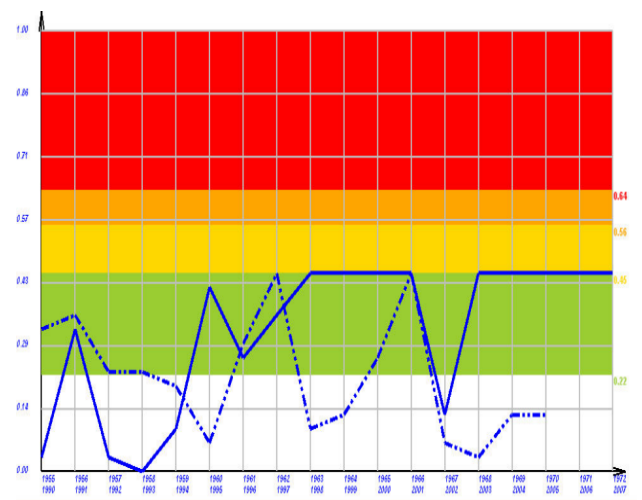

Figure 6: FEVs for Tbilisi in August for periods 19551970 (“--”line) and 1990-2007 (“-.-”line).

in another. We have done several experiments with data available from the Ministry of Preservation of the Environment of Georgia and have considered the change in FEVs due to different representation of fuzzy sets.

On Figures 7 it was shown how the FEVs differ if we change the parameters of trapezoidal fuzzy sets. Trapezoidal representation of fuzzy sets allow the certain flexibility to represent the opinion of experts in gradation of HIs.

In the previous work [16] we have considered the step-wise representation of fuzzy set as shown in Figure 8(a). The fuzzy set hot weather was separated into segments, and each segment had a linguistic name (normal, warm, very warm, hot, very hot, extremely hot) and was represented by different colours (Figure 8). As a result we have found the FEV for the fuzzy set hot weather, each months and the result was also get into one on the above mentioned regions (green, yellow, orange, red). Collecting results of FEVs of each month, say August, (see for example Figure 6) for the main city of Georgia Tbilisi and some regions, we have analyzed the peaks and plateaus with respect to possible heat waves. In this work we represent separated fuzzy sets normal, warm, very warm, hot, very hot, extremely hot, find the FEV for each fuzzy set and then we do the aggregation of these values to obtain one FEV that will be a characteristics of HIs within the corresponding period of time. The task of the appropriate aggregation of FEVs is still under investigation, but currently we consider the weights 

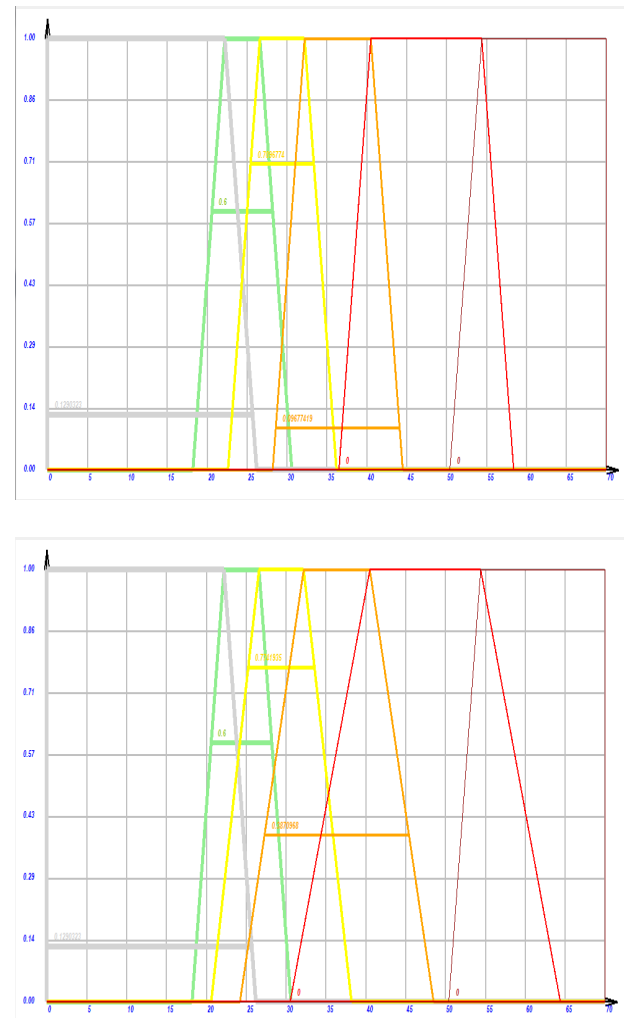

Figure 7: Different representation of fuzzy sets and their corresponding FEVs.

for each FEV for normal, warm, very warm, hot, very hot, extremely hot to highlight "the more warm" characteristics. For example if the FEV for hot is 0.4 and the FEV for normal is 0.6 we give the priority to FEV for hot.

\section{A comparison with other approaches and methodologies}

Although the importance of a problem for better understanding of HWs was already recognized long time ago [8], [12], the methods for investigation are still disputable. Analyzing the HW on a daily basis can reveal how fast people responded to the conditions, and that identifying the magnitude of an event by daily intensity would be necessary.

Predictive models, based on the empirical-statistical models allow exploration of HW-disease relationship in time (e.g., monthly temperature and food-poisoning in a population); to estimate change in temperature-related cases under future climate change [24]. Qualitative or quantitative measurements describe basic climate-health relationships, e.g. correlation of interannual variation in malaria incidence with minimum September temperature; analogue of a warming trend, e.g. association of changes in malaria incidence in highland areas with a trend in temperature; impacts of extreme event, e.g. assessment of mortality associated with a heatwave; geographical analogue, e.g. comparison of vector activity in two locations, the second location having a climate

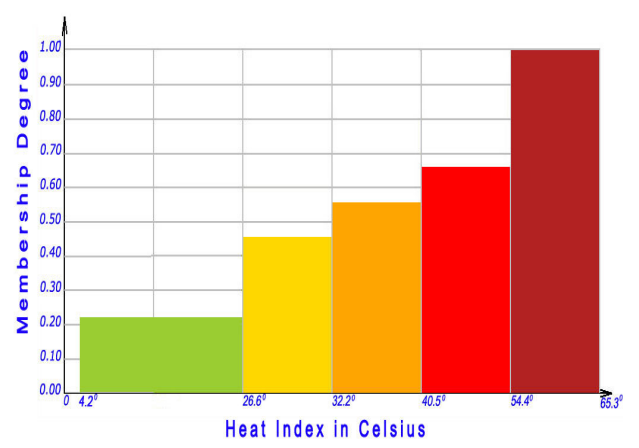

(a) Fuzzy set hot weather

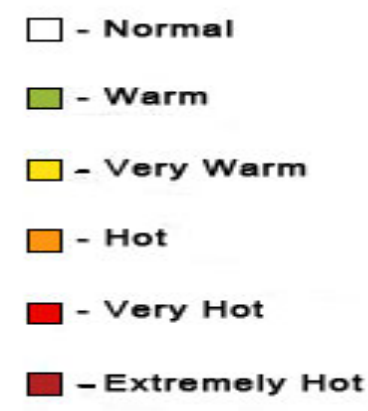

(b) The legend for hot weather

Figure 8: A step-wise function representing the fuzzy set hot weather.

today that is similar to that forecast for the first location [9].

Most approaches are based on the simple definitions of HW as an event of high HI during several days [1], [3], [6], [13], [15], [21], [25], [26], [33], [36]. An intention of researches is to discover whether there is a trend in the number of HWs in different parts of the world and the duration of HWs over the past years and, if so, what the magnitude of the trend is [32]. But certainty that aspects of the future will not be direct extensions of the past or present, ensures that our knowledge base is incomplete for predicting the range, timing and magnitude of future health impacts of HWs. Uncertainties in modelling are therefore unavoidable, and explicit caveats must be stated [5, 22, 23]. Increasing scientific effort is underway to develop integrated mathematical modelling that takes account of many of these aspects. In [7] uncertainty in the representation of HWs is captured by interval, where different categories of HWs are defined. But intervals are defined rigorously, and the natural question arises (see Table 1): if strong heat stress is estimated by $\geq 120$ units, what about 119 units?

To the best of our knowledge, fuzzy methods for HWs investigations were not yet applied. From this point of view our approach can be considered as an innovative one in the definition and investigation of the HWs. FEV approach is chosen due to its appropriateness for the available real data as well as its awareness in capturing the subjectiveness in the description of HWs.

Some remarks can be still done concerning the ap- 
plied methods.

Investigation of HWs in our approach are based on the notion of HIs. We do not restrict the duration of HW to a particular number of days with high HIs. Contrarily to the approach in [7], for example, the user of our software decides him/herself what period of time he/she wants to investigate with respect to HWs, such that the questions "was it very hot during July-August 1969 in Tbilisi?" or "was it hot during Jul 1958, first week, in Poti?" are eligible.

In our work we deal with linguistic description [37] of the HIs and HWs. In the the representation of the fuzzy sets normal, warm, very warm, hot, very hot, extremely hot we relay on the opinion of the experts [34], on the common sense as well as availability of the data Due to the recent developed methodologies (see, e. g., $[17,18,19,20])$ this approach can be further modified and formalised, and the opinion of the different experts, concerning the estimation of the hot weather in the particular region of Georgia can be constructed. In this paper fuzzy sets describing different characteristics of a HW are represented as trapezoidal fuzzy sets, and an expected values for each fuzzy set is calculated, the accumulated information based on. e.g., OWA (Ordered Weighted Averaging) operator (and those operators, belonging to its family[19]) can help us to classify the HWs into different categories (e.g., mild, moderate, strong, severe, extreme). In our work we have chosen these categories to distinguish HW from HI, to show what the relationships between these two notions exist and how they can be classified.

\section{Conclusions and future work}

The paper concerns influences of the global warming on a climate of Georgia and health of the population. In particular, one important parameter of a human thermal comfort - a heat index (which represents a combination of the air temperature and relative humidity) - has been investigated and its connection to $\mathrm{HW}$ is established.

The research has been done in close cooperation with the Ministry of Preservation of the Environment of Georgia. The data - temperature and relative humiditywere available. The received information has been classified and ranged, and on its base the software has been created (by means of $\mathrm{C} \#$ ). The software enables to see both numerical and graphical representation of the HIs, and also the following operations are possible:

- by means of the radio-buttons, allocated on the map of Georgia, it is possible to select an interesting city in Georgia (Tbilisi, Poti, Lentekhi and Dedoplistskharo);

- during input of daily average temperature and relative humidity, $\mathrm{HI}$ is automatically calculated;

- it is possible to consider a graphical image of a daily average air temperature, a relative humidity and HI of each month, each year and each period;

- to define and count up HIs for an appropriate interval (a month, or other periods);
- graphically view the tendency of HIs of each month for the first and second period for the purpose of forecasting;

- to select the maximum and the minimum value of temperatures, relative humidity and HI of a day, a month, a year and other periods;

- some other functions.

On the base of HIs we propose a new approach to define and investigate HWs. Definition of HWs is based on the most typical fuzzy expected values. Relaying on the search of the appropriate scientific literature we have revealed that fuzzy methods for HWs' investigations were not yet applied. From this point of view our approach can be considered as an innovative one in the definition and investigation of HWs. FEV approach is chosen due to its appropriateness for the available real data, as well as their awareness in capturing the subjectiveness in the description of the HWs.

FEVs are the numerical characteristics of the fuzzy sets. Using these values for one month, we can see if this month was hot or not. If it was hot then we conclude the availability of a strong HW during this month.

The software and methods for estimation of the results have been used in the preparation of the Second Communication of Georgia to the United Nations Framework Convention on Climate Change.

A variety of methods are required to assess the potential impacts of HWs on human health, including predictive modelling and expert judgement. The results presented in the paper reveal another (not applied yet) streams for future research: on the base of numerical data available to build fuzzy rules describing the influence of the HW on the human health in Georgia.

\section{References}

[1] Goldfields-Eucla Western Australia:climatic survey April 2000/Bureau of Meteorology, Department of the Environment and Heritage. http://catalogue.nla.gov.au/Record/997793.

[2] Health and Global Environmental Change SERIES, No. 2. Heat-waves: risks and responses. World Health Organization 2004. http://www.euro.who.int/.

[3] National weather service.http://www.weather.gov/.

[4] T. Holt and J. Palutikof. The effect of global warming on heat waves and cold spells in the Mediterranean. Climatic Research Unit, University of East Anglia, Norwich, UK. prudence.dmi.dk/public/publications/D5A4.doc.

[5] Malik Shahzad Kaleem Awan and Mian Muhammad Awais. Predicting weather events using fuzzy rule based system. Appl. Soft Comput., 11(1):5663, January 2011.

[6] H.S. Belding and T.F. Hatch. Index for evaluating heat stress in terms of resulting physiological strains. Heating, Piping Air Conditioning, 27:129_ 136, 1955. 
[7] E. H. Bowles. Classifying Heat Waves in the United States. $\mathrm{PhD}$ thesis, Kansas State University, Manhattan, Kansas, USA, 2009.

[8] A. Burrows. Hot waves: Conditions which produce them, and their effect on agriculture. In Yearbook of the U. S. Department of Agriculture, volume 22, pages 325-336. 1900.

[9] J. F. Feenstra, I. Burton, J. B. Smith, and R. S. J. Tol, editors. Handbook on Methods for Climate Change Impact Assessment and Adaptation Strategies. United Nations Environment Programme, vrije Universiteit Amsterdam, Institute for Environmental Studies, 1998.

[10] M. Grabisch. Fuzzy pattern recognition by fuzzy integrals and fuzzy rules. In S.K. Pal and A. Pal, editors, Pattern Recognition - From Classical to Modern Approaches, pages 257-280. World Scientific, 2002.

[11] M. Grabisch, H.T. Nguyen, and E.A. Walker. Fundamentals of Uncertainty Calculi, with Applications to Fuzzy Inference. Kluwer Academic, 1995.

[12] F. Houghton and C. Yaglou. Determining equal comfort lines. Journal of the American Society of Heating and Ventilating Engineers, 29:165-176, 1923.

[13] L. Kalkstein, J. Greene, D. Mills, A. Perrin, and et al. Analog european heat waves for u.s. cities to analyze impacts on heat-related mortality. Bulletin of the American Meteorological Society, 89(1):7585, 2008.

[14] A. Kandel. Fuzzy expert systems. CRC Press, Inc, 2000 Corporate Blvd., N.W., Boca Raton, Florida 33431, 1991.

[15] T. Karl and R. Quayle. The 1980 heat wave and drought in historical perspective. Monthly Weather Review, 109(10):2055-2073, 1981.

[16] T. Kiseliova, M. Fandoeva, and A. Sikharulidze. Investigation of heat waves with fuzzy methods. Applied Soft Computing, Submitted 2012.

[17] Deng-Feng Li. Fuzzy linear programming approach to multiattribute decision making with linguistic variables and incomplete information. Advances in Complex Systems, 10(4):505-525, 2007.

[18] Deng-Feng Li. Multiattribute group decision making method using extended linguistic variables. International Journal of Uncertainty, Fuzziness and Knowledge-Based Systems, 17(6):793-806, 2009.

[19] Deng-Feng Li. Owa-based nonlinear mathematical programming approach to fuzzy multiattribute group decision making with linguistic variables. New Mathematics and Natural Computation, 6(3):285-300, 2010.

[20] Deng-Feng Li and Tao Sun. Fuzzy linmap method for multiattribute group decision making with linguistic variables and incomplete information. International Journal of Uncertainty, Fuzziness and Knowledge-Based Systems, 15(2):153-173, 2007.

[21] R. Livezey and R. Tinker. Some meteorological, climatological, and microclimatological considerations of the severe U.S. heat wave of mid-July
1995. Bulletin of the American Meteorological Society, 77(9):2043-2053, 1996.

[22] I. Maqsood and A. Abraham. Weather analysis using ensemble of connectionist learning paradigms. Applied Soft Computing, (7):995-1004, 2007.

[23] A. J. McMichael, A. Haines, and R. S. Kovats. Methods to assess the effects of climate change on health. In Elliot and et al [24], pages 55-69.

[24] A.J. McMichael, W.J.M. Martens, R. S. Kovats, and S. Lele. Climate change and human health: mapping and modelling future impacts. In P. Elliot and et al, editors, Disease exposure and mapping. Oxford University Press.

[25] J. 1991 Oliver. The history, status and future of climatic classification. Physical Geography, 12(3):231-251, Oliver, J. 1991.

[26] R. Quayle and F. Doehring. Heat stress: A comparison of indices. Weatherwise, (34):120-124, 1981.

[27] P. Robinson. On the definition of a heat wave. Journal of Applied Meteorology, 40:762-775, 2001.

[28] G. Sirbiladze and A. Sikharulidze. Weighted fuzzy averages in fuzzy environment. Part I: Insufficient expert data and fuzzy averages. International Journal of Uncertainty, Fuzziness and KnowledgeBased Systems, 11(2):139-157, 2002.

[29] R. G. Steadman. The assessment of sultriness. Part I: A temperature-humidity index based on human physiology and clothing science. Journal Applied Meteorology, 18(7):861-873, 1979.

[30] R. G. Steadman. A universal scale of apparent temperature. Journal of Applied Meteorology, 23(12):1674-1687, 1984.

[31] M. Sugeno. Theory of fuzzy integrals and its applications. PhD thesis, Tokyo Institute of Technology, 1974.

[32] A. Tamrazian, S. LaDochy, J. Willis, and W.C. Patzert. Heat waves in southern california: Are they becoming more frequent and longer lasting? APCG YEARBOOK, 70:59-69, 2008. DOI: 10.1353/pcg.0.0001.

[33] E. Thom. The discomfort index. Weatherwise, pages 57-60, 1959.

[34] I. Vachiberidze. Boomerang Principle. Climate Change and Health Care (in Georgian). Second National Communication to the UNFCCC, Tbilisi, 2008.

[35] S. Vassiliadis, G. Triantafyllos, and G. G. Pechanek. A method for computing the most typical fuzzy expected value. In Proceedings of the Third IEEE Conference on Fuzzy Systems, pages 2040-2045, June 1994.

[36] O. Wilhelmi, K. Purvis, and R. Harriss. Designing a geospatial information infrastructure for mitigation of heat wave hazards in urban areas. Natural Hazards Review, pages 147-158, 2004.

[37] L. A. Zadeh. Toward a generalized theory of uncertainty (GTU)-an outline. Inform. Sci., 172:1-40, 2005. 